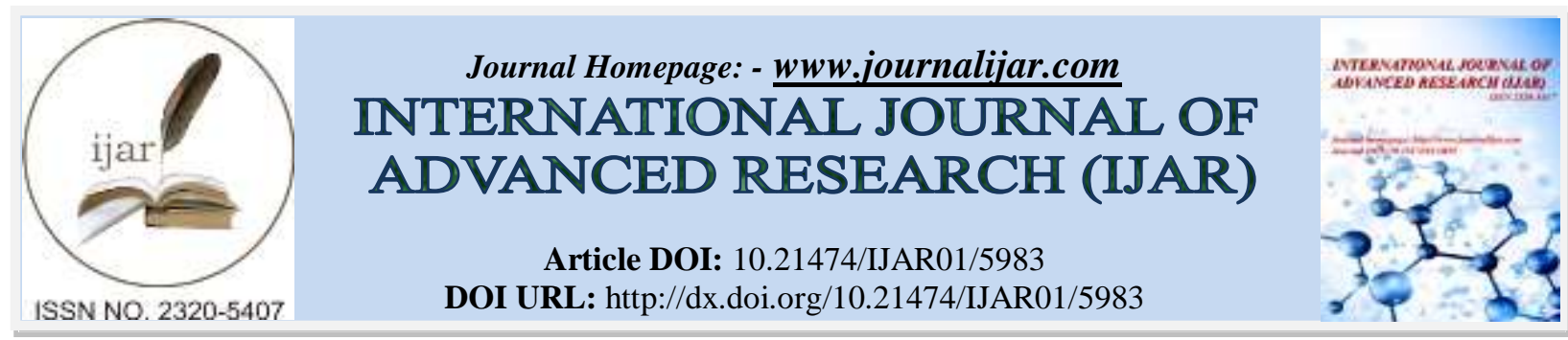

RESEARCH ARTICLE

\title{
ROSE BENGAL STAIN AS A DIAGNOSTIC AID IN DETECTING ORAL POTENTIALLY MALIGNANT LESIONS AMONG TOBACCO CHEWERS - A HOSPITAL BASED STUDY.
}

Dr. Pushpa ${ }^{1}$, Dr. Vaishali Keluskar ${ }^{2}$, Dr. Anjana Bagewadi ${ }^{3}$ and Dr. Alka Kale ${ }^{4}$.

1. Post graduate, MDS in Oral Medicine and Radiology, KAHER KLE V.K. Institute of Dental Sciences, Belagavi, Karnataka, India.

2. Professor, Department of Oral Medicine and Radiology, KAHER KLE V.K. Institute of Dental Sciences, Belagavi, Karnataka, India.

3. Head and Professor, Department of Oral Medicine and Radiology, KAHER KLE V.K. Institute of Dental Sciences, Belagavi, Karnataka, India.

4. Principal and Professor, Department of Oral Pathology, KAHER KLE V.K. Institute of Dental Sciences, Belagavi, Karnataka, India.

\section{Manuscript Info}

Manuscript History

Received: 06 October 2017

Final Accepted: 08 November 2017

Published: December 2017

Key words:-

Oral potentially malignant lesion(OPL),

Rose Bengal stain (RB).

\section{Abstract}

Background: Oral cancer is a major public health problem in India usually preceded by precancerous lesions. Among all diagnostic aids, vital staining is simple, inexpensive and sensitive tool for identifying epithelial dysplasia and can be used for screening. Hence in the present study an attempt was made to evaluate efficacy of RB stain in early detection of oral potentially malignant among tobacco chewers and correlate the intensity of Rose Bengal stain with the degree of dysplasia.

Objectives: To determine histopathological changes in biopsy specimens taken from tobacco chewers with positive and negative stain.

To correlate intensity of the stain clinically with histopathological features taken from tobacco chewers.

Methodology: The study comprised of 105 subjects with tobacco chewing habit for more than a year. RB stain was applied in the area of tobacco placement. Based on areas of highest uptake of stain, incisional biopsy was done. The intensity of uptake of stain was compared with the histological findings.

Statistical analysis: One-way analysis of variance (ANOVA) test was applied.

Results: RB stain was taken up in all tobacco chewers with or without lesions. Sensitivity was $93.33 \%$

Conclusion: RB stain is simple, cost- effective, non- allergic and is not taken up by inflammatory cells unlike Toluidine Blue stain. Sensitivity of RB staining method was found to be higher when compared with Toluidine Blue staining. Thus RB stain can be used as a diagnostic test in screening of potentially malignant lesions.

Copy Right, IJAR, 2017,. All rights reserved.
Corresponding Author:- Pushpa.

Address:- Post graduate, MDS in Oral Medicine and Radiology, KAHER KLE V.K. Institute of Dental Sciences. Belagavi. Karnataka. India. 


\section{Introduction:-}

Oral cancer $(\mathrm{OC})$ is one of the most prevalent cancer which ranks sixth in the global perspective of cancer burden with the highest mortality rate. ${ }^{1}$ Oral cancer is often preceded by oral potentially malignant lesions (OPLs). Emphasis has been placed on early detection and diagnosis of potentially malignant and malignant disorders. $^{2}$

Early detection of oral precancerous lesions is still a diagnostic challenge for most of the clinicians and ideal adjuncts for detections are currently unavailable.

Among all diagnostic aids, vital staining is simple, inexpensive and sensitive tool for identifying epithelial dysplasia and early stage of carcinoma and can be used for screening large population. Vital staining like toluidine blue and Lugol's iodine has been used previously for detection of premalignant and malignant lesions, but studies have often revealed false positive results. ${ }^{2,3}$

Rose Bengal (RB) dye is 4, 5, 6, 7 tetrachloro-2, 4, 5, 7 tetraiododerivate of fluorescein. ${ }^{2,3}$ Literature has reported very few studies regarding the efficacy of Rose Bengal stain in detecting premalignant and malignant disorders.

Hence in the present study an attempt was made to evaluate efficacy of RB stain in early detection of oral potentially malignant lesions.

Aim:-

To evaluate the efficacy of Rose Bengal stain among tobacco chewers for identification of dysplastic areas clinically and correlating it with the histological grading of dysplasia.

\section{Objectives:-}

To detect the retention of Rose Bengal stain clinically among tobacco chewers.

To determine histopathological changes in biopsy specimens obtained from tobacco chewers with positive and negative staining.

$\square$ To correlate intensity of the stain clinically with histopathological findings obtained from tobacco chewers.

\section{Methodology:-}

\section{Source of data}

Study sample included 105 subjects with tobacco chewing habit reporting to the Department of Oral Medicine and Radiology, K.L.E Vishwanath Katti Institute of Dental Sciences, Belagavi, Karnataka with an informed consent. The study was approved by the Institutional Ethical and Research committee of K.L.E Vishwanath Katti Institute of Dental Sciences, Belgavi. The subjects were then interviewed in person regarding duration and frequency of tobacco chewing.

\section{Method Of Collection Of Data:-}

A total of 105 tobacco chewers were included in this study.

\section{Inclusion criteria:-}

Patients with habit of tobacco chewing for more than one year.

\section{Exclusion criteria:-}

- Patients who were smokers and alcoholics.

- $\quad$ Patients previously diagnosed/ treated with potentially malignant disorders.

- Patients with frank malignancy.

\section{Armamentarium}

1. Clinical examination of the patient

2. Rose Bengal staining

a. $1 \%$ Rose Bengal stain 
b. Cotton applicator

c. Distilled water

d. 4 grades of Rose Bengal stain (shade guide)

3. For comparing clinical picture with grading system a. Adobe photoshop CS3

\section{Case History:-}

A detailed history with thorough clinical examination was done and findings were recorded in a case history proforma. An informed consent was obtained after explaining entire procedure of the study to the patients.

\section{Procedure:-}

$\square \quad$ After a detailed case history and thorough clinical examination, area of tobacco quid placement was selected for staining.

$\square \quad$ Patients were asked to rinse their mouth with distilled water for 1 minute to clean any debris.

$\square \quad 1 \%$ Rose Bengal solution was applied with a cotton tip for 2 minutes.

- $\quad$ Patients were asked to rinse their mouth for 1 minute with distilled water to remove excess of Rose Bengal stain.

- $\quad$ Retention of the dye was recorded clinically and photographically using digital camera.

- $\quad$ After this, biopsy was performed under local infiltration.

- After fixation of the biopsy specimen, the slide was examined histopathologically and graded for dysplasia as:

O Grade I- Hyperkeratosis

O Grade II - Mild dysplasia

O Grade III - Moderate dysplasia

O Grade IV - Severe dysplasia

$\square \quad$ The intensity of Rose Bengal staining clinically was correlated with the microscopic changes using ADOBE PHOTOSHOP CS3

\section{Photograhs}

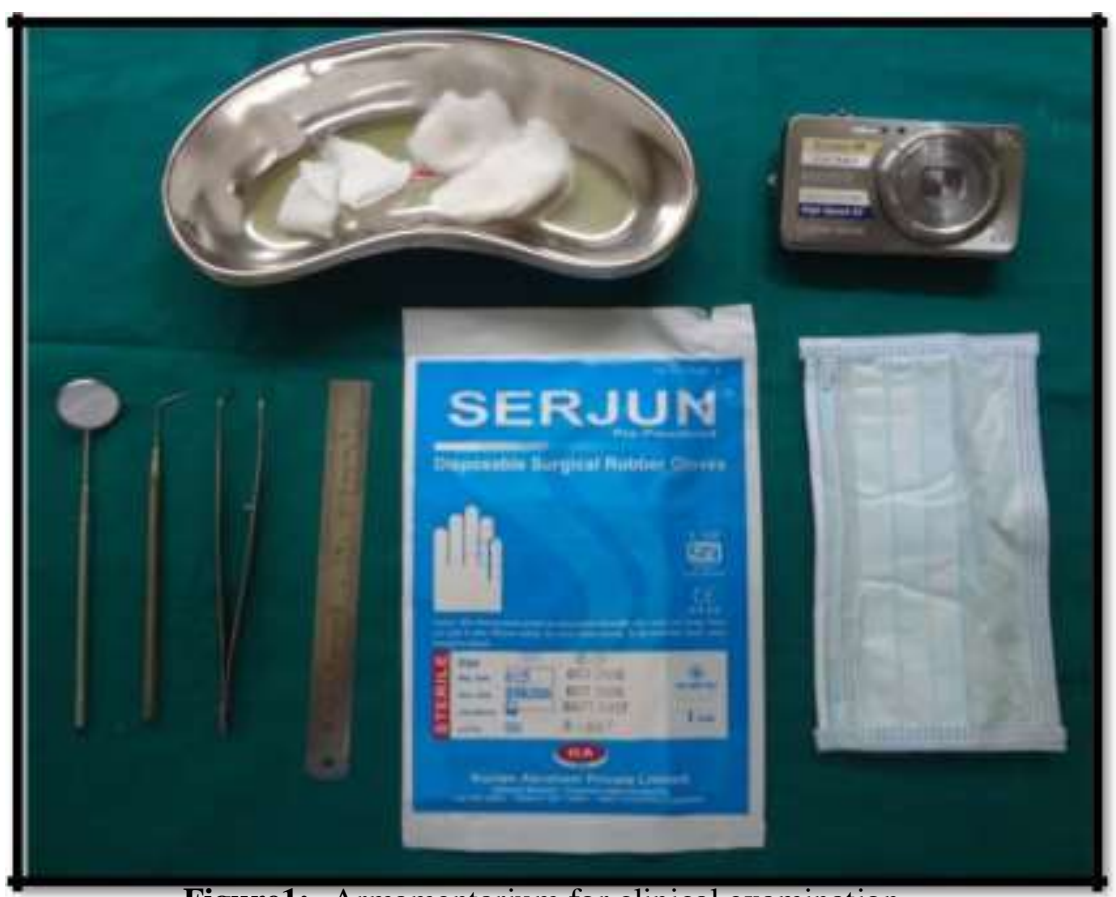

Figure1:- Armamentarium for clinical examination 


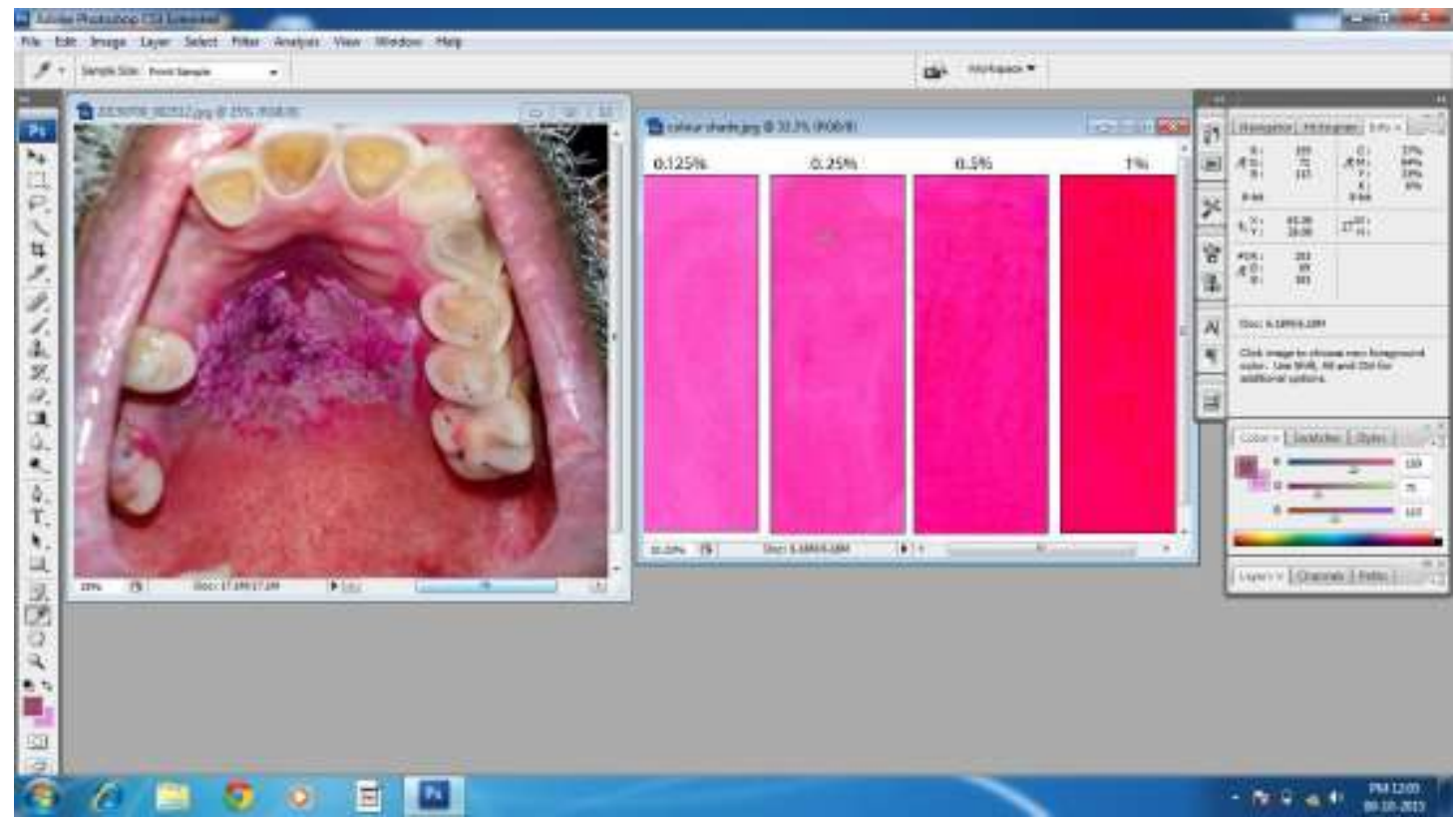

Figure2:- Intensity of Rose Bengal stain estimation using Adobe Software CS3
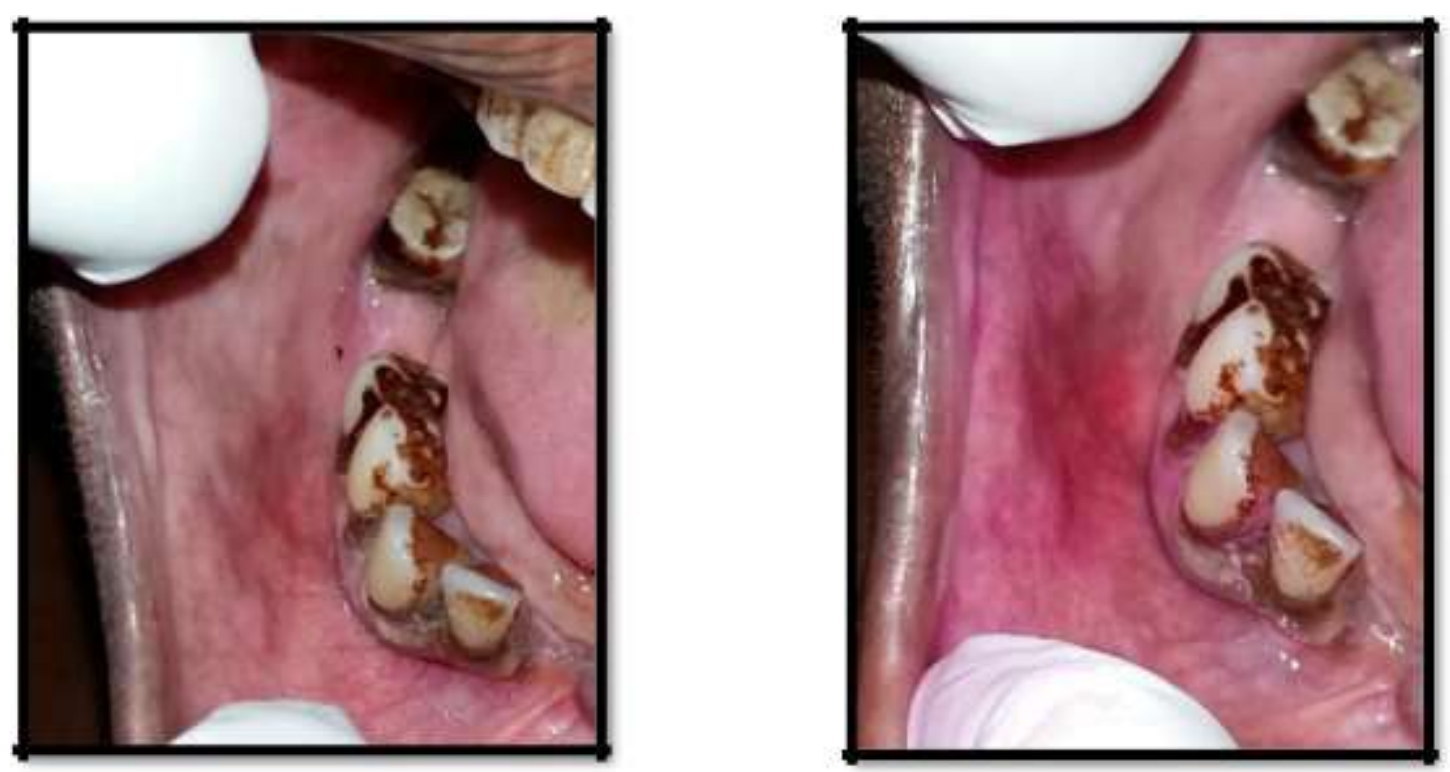

Figure 3:- Clinically apparent. 
Figure 4:- After staining mucosa at tobacco quid placement (Before staining).

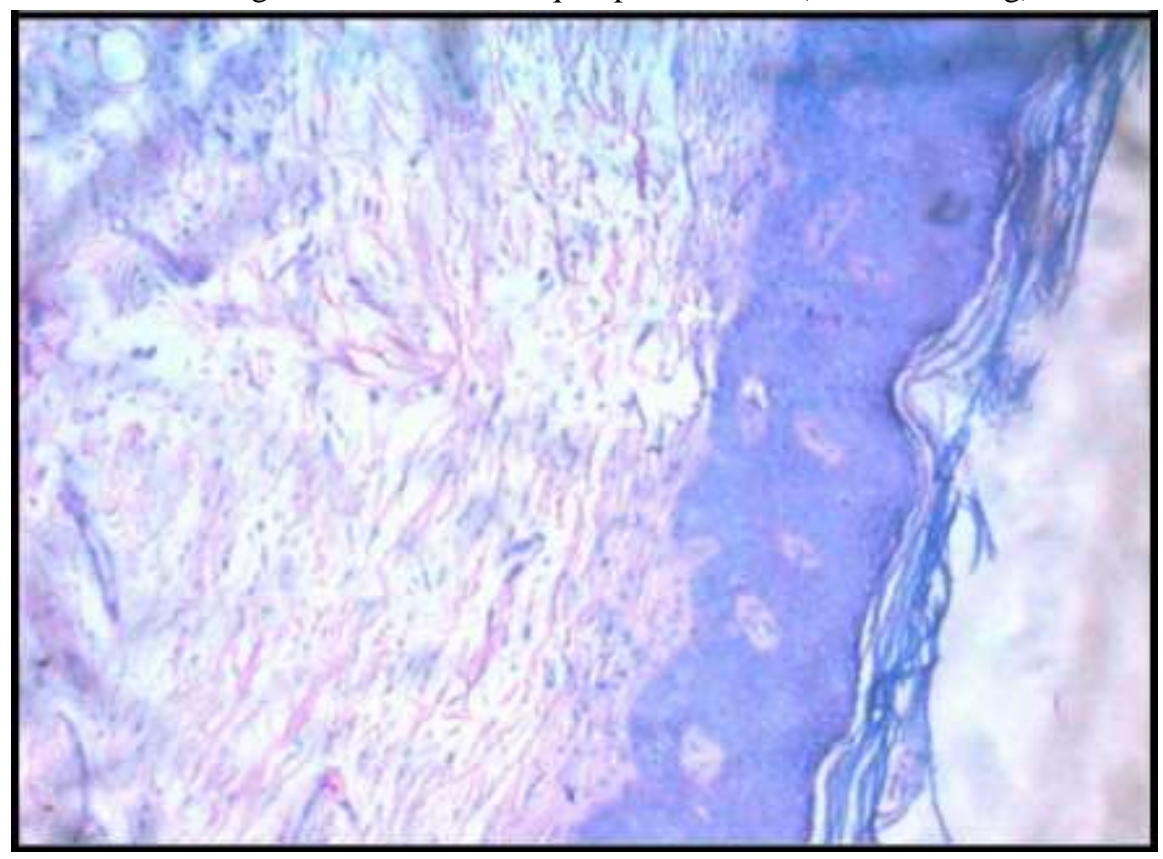

Figure 5:- Photomicrographs showing hyperkeratosis

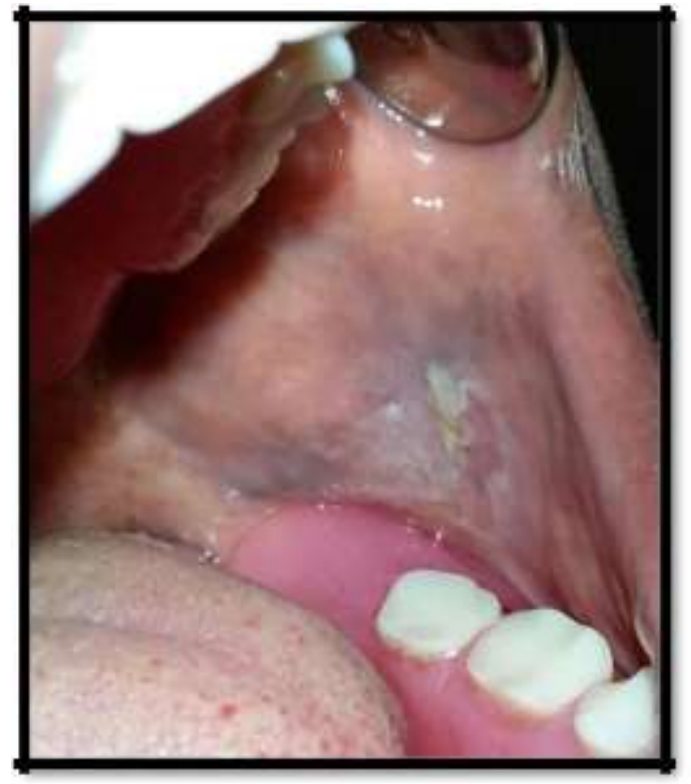

Figure 6: Clinically diagnosed- Tobacco induced keratosis (Before staining)

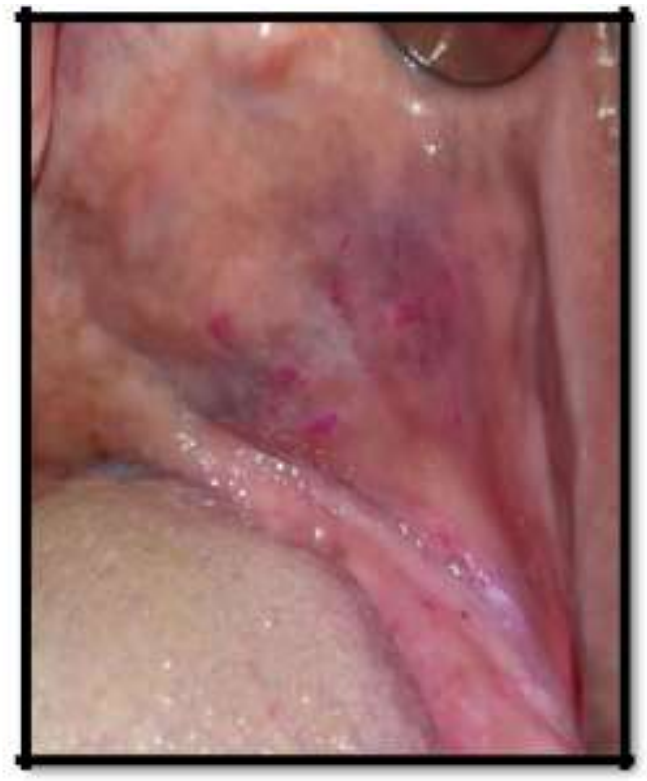

Figure 7: After staining 


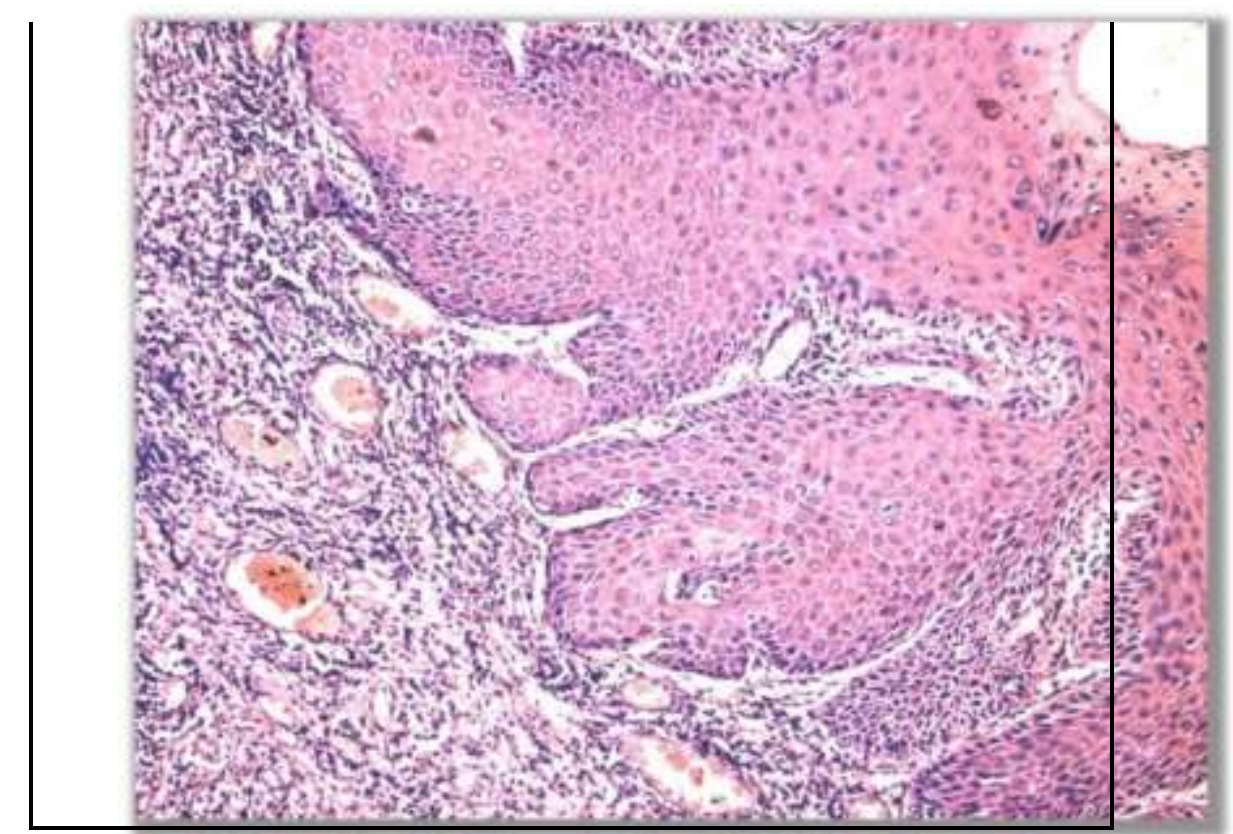

Figure 8:- Photomicrographs showing hyperkeratosis with mild dysplasia

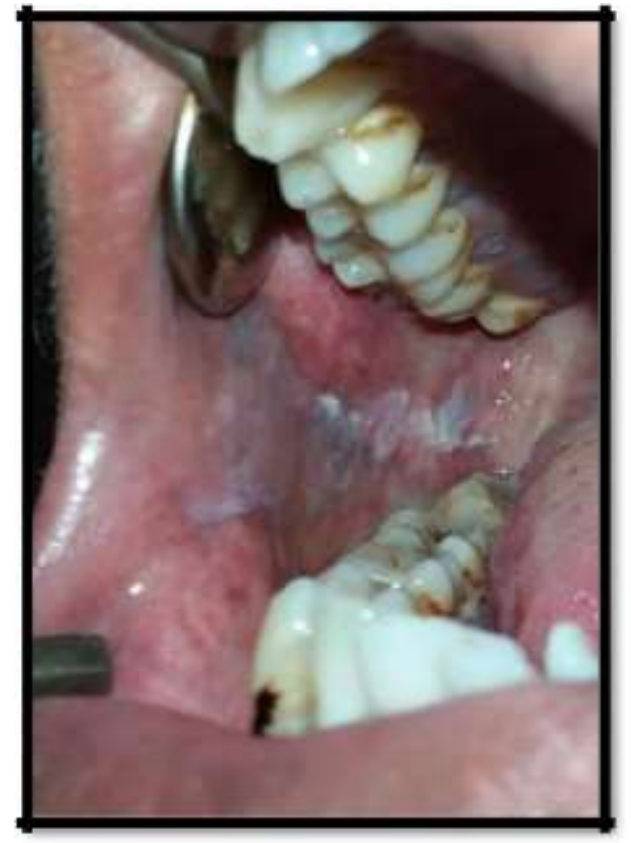

Figure 9: Clinically

diagnosed - Homogenous

Leukoplakia(Before staining)

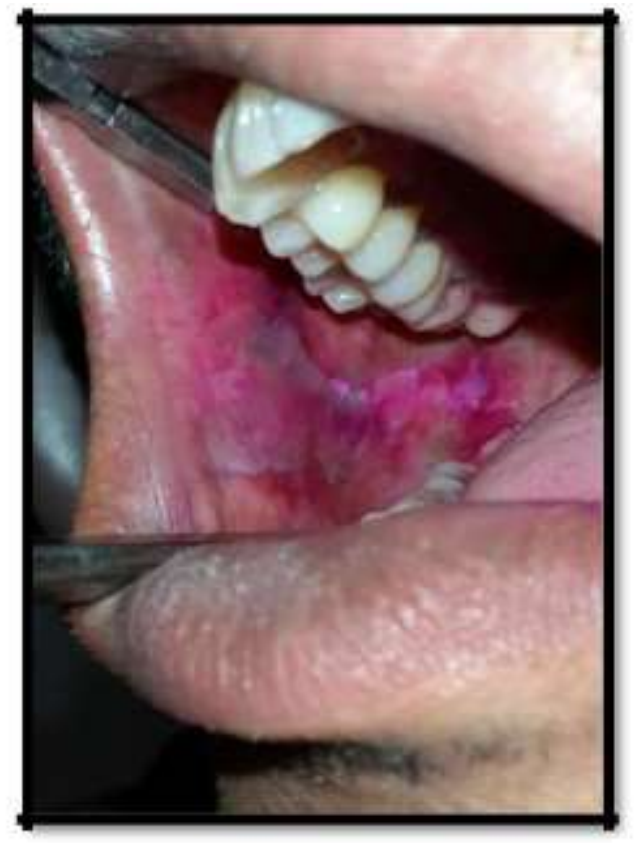

Figure 10: After staining 


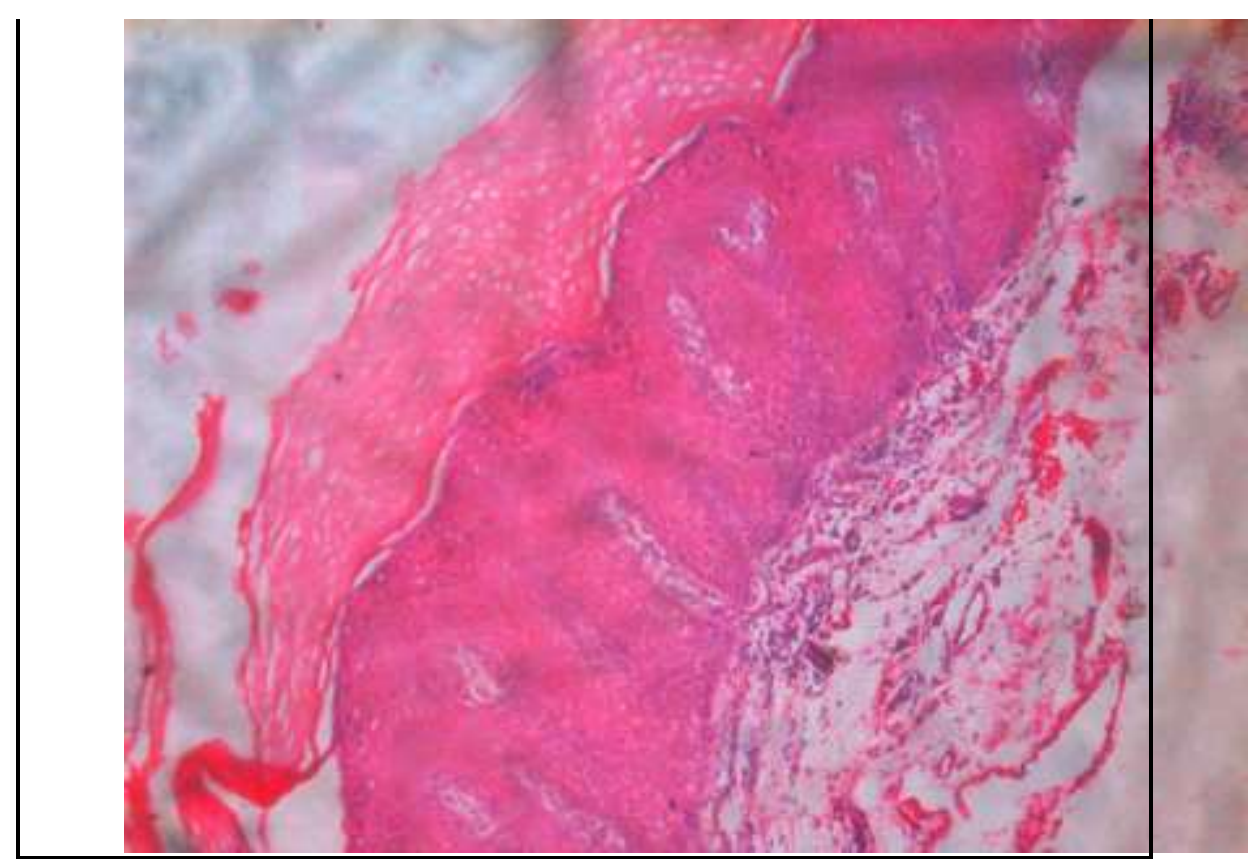

Figure 11:- Photomicrographs showing mild dysplasia

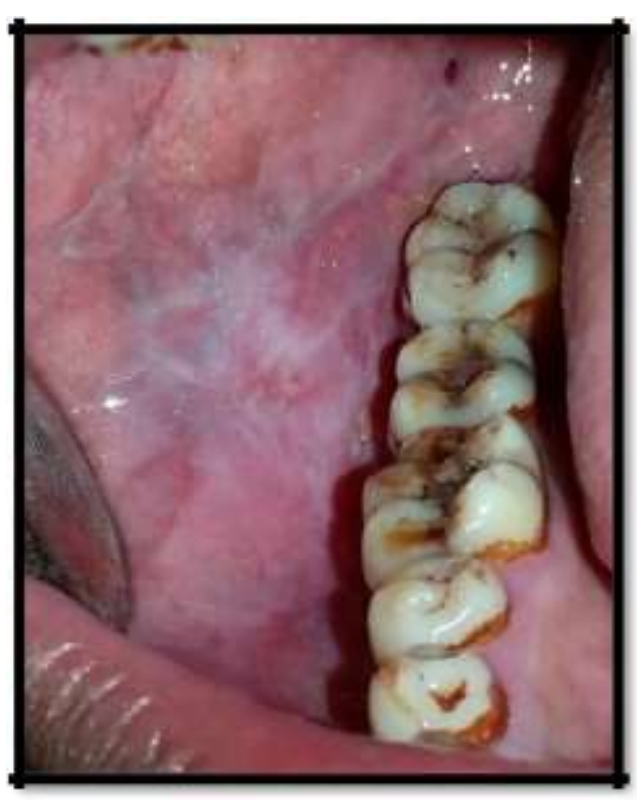

Figure 12: Clinically

diagnosed - Tobacco induced Lichenoid reaction (Before

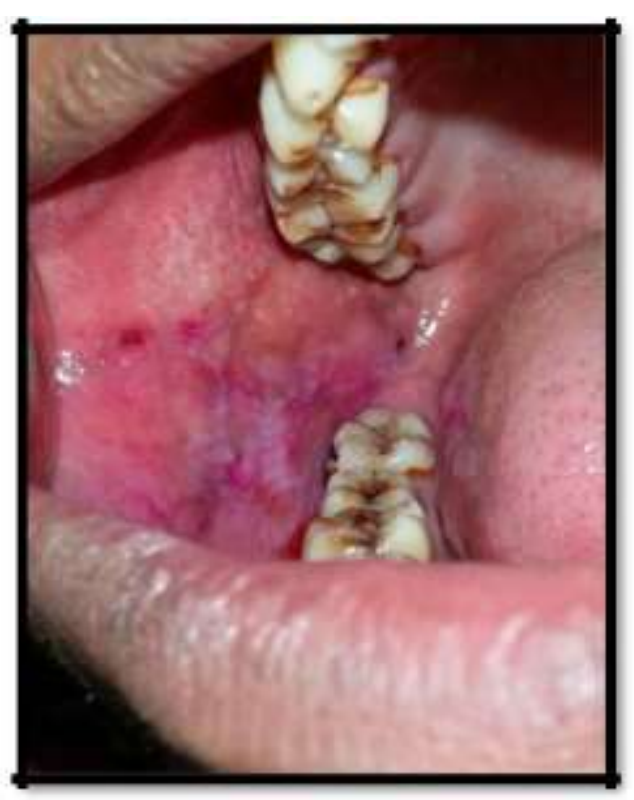

Figure 13: After staining

staining) 


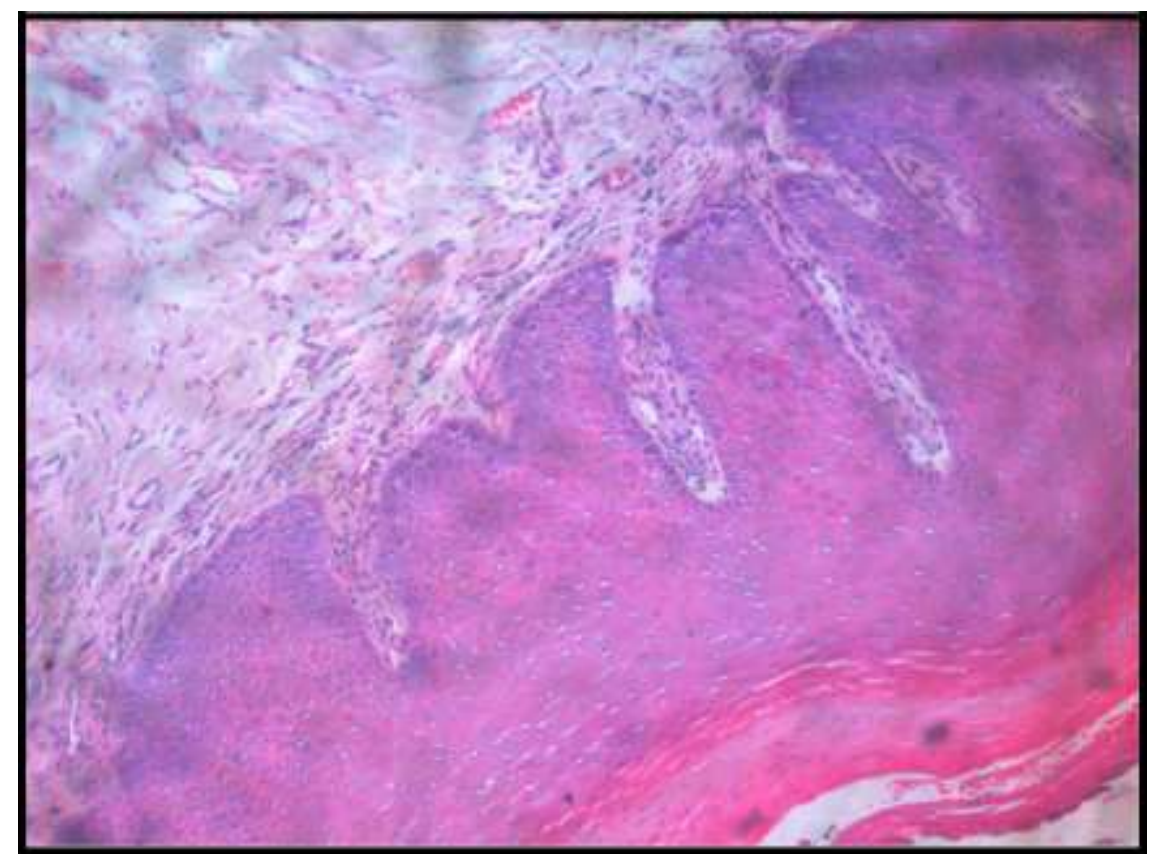

Figure 14:- Photomicrographs showing moderate dysplasia

\section{Statistical Analysis:-}

Spearman's correlation coefficient method was used for correlation between clinical and histopathological findings and these with stain scores.

Kappa statistics was applied to analyse agreement between clinical diagnosis and histopathological findings.

One way ANOVA was applied to compare grades of histopathological findings with mean duration and frequency of tobacco chewing and with stain scores.

One way ANOVA was applied to compare grades of clinical findings with mean duration and frequency of tobacco chewing and stain scores.

Pair wise Tukeys multiple posthoc procedures was used to compare between grades of clinical findings and histological findings.

Karl's Pearson's correlation coefficient method was applied to correlate between duration and frequency with stain scores.

To calculate sensitivity, specificity, positive predictive values(PPV), negative predictive values(NPV) of Rose Bengal stain in detecting oral premalignant lesions.

\section{Results And Observation:-}

The present study included 105 patients who had habit of tobacco chewing for more than 1 year. This study was undertaken among tobacco chewers to correlate the intensity of RB stain with clinical findings and histopathological features.

The mean age of all the patients which was 41.67. These results indicate that there was no significant age difference between the patient. Among them 95 patients were males and 10 patients were females. The mean age of male which was 40.37 and mean age of female was 54 years.

(Table 1) Correlation between clinical diagnosis with histo-pathological diagnosis by Spearman's correlation coefficient method shows significant $\mathrm{p}$ - value among all patients. 
(Table 2) Correlation between clinical diagnosis with stain scores by Spearman's correlation coefficient method shows significant $\mathrm{p}$-value which was $0.0022^{*}$

(Table 3) Correlation between histo-pathological diagnosis with stain scores by Spearman's correlation coefficient method shows highly significant p-value which was $0.00001 *$

Score 0 - no lesion but H/o habit chewing

Score1- Tobacco induced keratosis

Score2- Leukoplakia

Score3- Erythroplakia

Score4- Others- OLP/ OLR/ OSMF/ Verrucous Leukoplakia

Histo-pathological diagnosis -

Score 0- No stain

Score1- Hyperkeratosis

Score 2- Mild dysplasia

Score3- Moderate dysplasia

Score4- Severe dysplasia / OLP/ OLR/ OSMF

Among the study subjects, 7 patients had no lesion but positive H/o habit and revealed hyperkeratosis.

48 patients were clinically diagnosed with tobacco induced keratosis which histopathologically revealed hyperkeratosis in 44 patients and mild dysplasia in 4 patients.

26 patients were clinically diagnosed with homogenous leukoplakia which showed histopathologically as hyperkeratosis in 13 patients, mild dysplasia in 9 patients and moderate dysplasia in 4 patients.

8 patients were clinically diagnosed as erythroplakia which reveled moderate dysplasia histopathologically.

16 patients were clinically diagnosed as Oral Lichen Planus, Oral Lichenoid Reaction, Oral Submucous Fibrosis, Verrucous Leukoplakia which showed histologically as hyperkeratosis in 5 patients, mild dysplasia in 1 patient, moderate dysplasia in 2 patients and 8 patients revealed Oral Lichen Planus, Oral Lichenoid Reaction, Oral Submucous Fibrosis.

Kappa statistics was $88.81 \%$ which was in agreement between clinical diagnosis and histo-pathological diagnosis.

(Table 4) Specificity and sensitivity of clinical diagnosis over histo-pathological diagnosis, as stain was taken in all patients with or without lesions, therefore Sensitivity was $93.33 \%$ with PPV $=100$

Specificity was not applicable as stain was taken up in areas of tobacco quid placement with no clinically apparent lesion.

(Table 5) Comparison of grades of histopathology diagnosis with stain scores by one way ANOVA showed the intensity of staining was directly proportional with the grade of dysplasia with highly significant $p$-value of $0.00001^{*}$

Increase in severity of grades of dysplasia, there is definite increase in frequency and duration of tobacco chewing habit with p- value 0.018 which is statistically significant.

The pair wise comparison of groups revealed that frequency and duration of habit was significantly high among Group 1 when compared with Group 3.

(Table 6) Comparison of grades of clinical diagnosis with stain scores by one way ANOVA shows as the grade of clinical diagnosis increases in various forms of tobacco chewers, the clinical uptake of stain also increases with the significant p-value of 0.00001 * 
Table 1

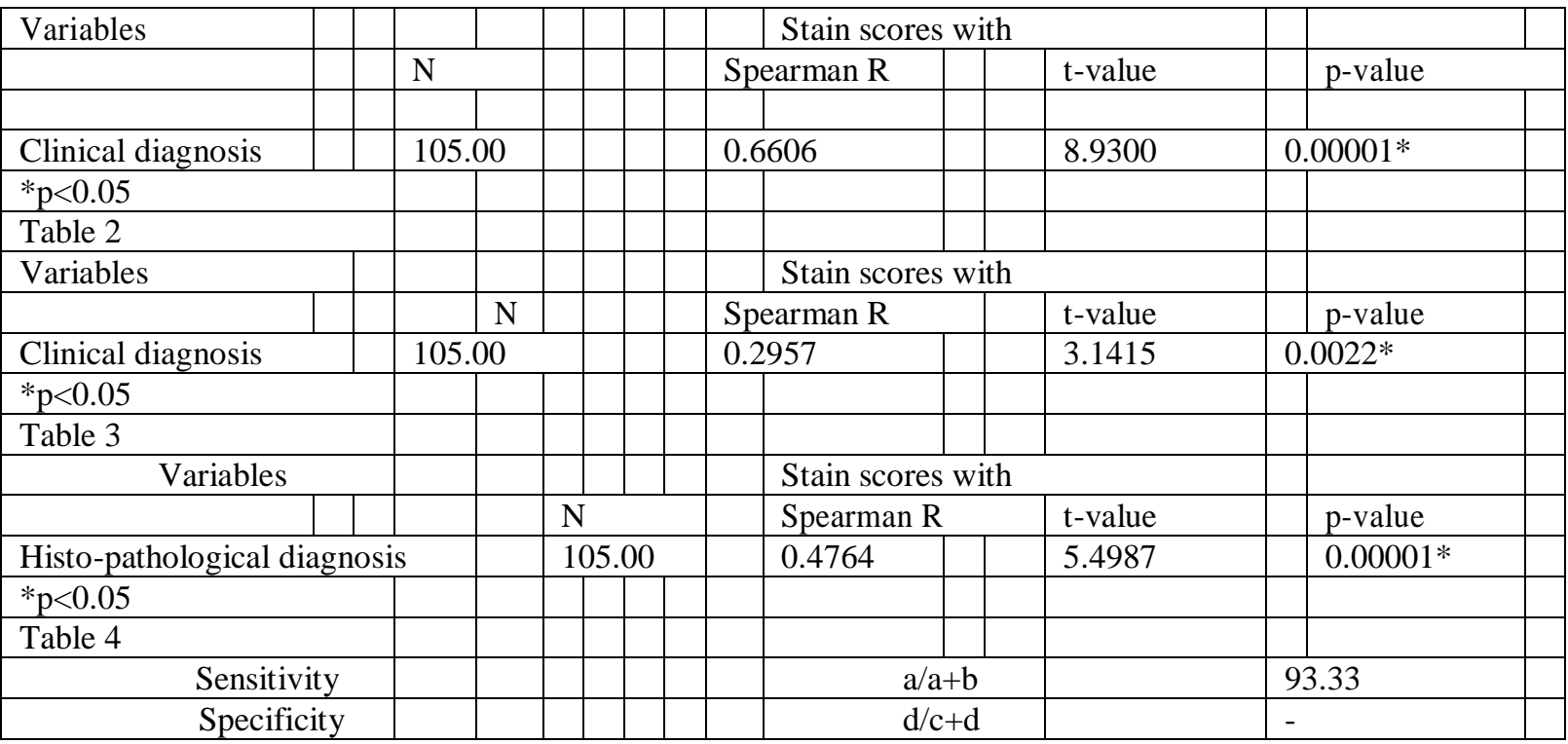

Table 5

\begin{tabular}{|l|l|l|}
\hline Positive predictive value & $\mathrm{a} / \mathrm{a}+\mathrm{c}$ & 100.00 \\
\hline Negative predictive value & $\mathrm{d} /(\mathrm{b}+\mathrm{d})$ & - \\
\hline
\end{tabular}

\begin{tabular}{|c|c|c|c|}
\hline Grades & & Stain $\mathrm{s}$ & cores \\
\hline & Mean & & SD \\
\hline Grade I & 77.12 & & 6.56 \\
\hline Grade II & 83.93 & & 5.25 \\
\hline Grade III & 88.71 & & 8.11 \\
\hline Others & 79.63 & & 10.01 \\
\hline F-value & & \multicolumn{2}{|c|}{12.8548} \\
\hline $\mathrm{p}$-value & & \multicolumn{2}{|l|}{$0.00001 *$} \\
\hline \multicolumn{4}{|c|}{ Pair wise comparisons by Tukeys multiple posthoc procedures } \\
\hline Grade I vs Grade II & & \multicolumn{2}{|c|}{$\mathrm{p}=0.0061 *$} \\
\hline Grade I vs Grade III & & \multicolumn{2}{|c|}{$\mathrm{p}=0.0001 *$} \\
\hline Grade I vs Others & & \multicolumn{2}{|c|}{$\mathrm{p}=0.7666$} \\
\hline Grade II vs Grade III & & \multicolumn{2}{|l|}{$\mathrm{p}=0.2660$} \\
\hline Grade II vs Others & & \multicolumn{2}{|l|}{$\mathrm{p}=0.5008$} \\
\hline Grade III vs Others & & \multicolumn{2}{|c|}{$\mathrm{p}=0.0197 *$} \\
\hline \multicolumn{4}{|l|}{$* \mathrm{p}<0.05$} \\
\hline \multicolumn{4}{|l|}{ Table 6} \\
\hline \multirow[t]{2}{*}{ Grades } & & \multicolumn{2}{|c|}{ Stain scores } \\
\hline & Mean & & SD \\
\hline Grade 0 & 79.57 & & 8.73 \\
\hline Grade I & 77.71 & & 4.95 \\
\hline Grade II & 80.62 & & 5.74 \\
\hline Grade III & 93.25 & & 2.31 \\
\hline Others & 77.88 & & 12.87 \\
\hline F-value & & \multicolumn{2}{|l|}{8.7487} \\
\hline p-value & & \multicolumn{2}{|l|}{$0.00001 *$} \\
\hline \multicolumn{4}{|c|}{ Pair wise comparisons by Tukeys multiple posthoc procedures } \\
\hline Grade 0 vs Grade I & & \multicolumn{2}{|l|}{$\mathrm{p}=0.9655$} \\
\hline Grade 0 vs Grade II & & \multicolumn{2}{|l|}{$\mathrm{p}=0.9969$} \\
\hline
\end{tabular}




\begin{tabular}{|l|l|l|}
\hline Grade 0 vs Grade III & & $\mathrm{p}=0.0028^{*}$ \\
\hline Grade 0 vs Others & & $\mathrm{p}=0.9839$ \\
\hline
\end{tabular}

\begin{tabular}{|l|l|}
\hline Grade I vs Grade II & $\mathrm{p}=0.4412$ \\
\hline Grade I vs Grade III & $\mathrm{p}=0.0001^{*}$ \\
\hline Grade I vs Others & $\mathrm{p}=0.9999$ \\
\hline Grade II vs Grade III & $\mathrm{p}=0.0003^{*}$ \\
\hline Grade II vs Others & $\mathrm{p}=0.7368$ \\
\hline Grade III vs Others & $\mathrm{p}=0.0001^{*}$ \\
\hline$* \mathrm{p}<0.05$ & \\
\hline
\end{tabular}

\section{Discussion:-}

Oral cancer (OC) is a major health problem particularly in developing countries. There is a global need to explore new techniques for the early prediction of OC. Most of the screening methods available are invasive, expensive and not feasible. There are various vital stains available to determine the lesions which are susceptible to OC. Rose Bengal dye is one staining dye which is diluted in distilled water to make upto $1 \%$ concentration.

Thus in the present study, we investigated the efficacy of Rose Bengal stain in detection of oral precancerous lesions. Study included 105 patients with the history of tobacco chewing habit of more than a year who fulfilled the inclusion and exclusion criteria. These patients were clinically evaluated and history of duration, frequency and placement of tobacco quid was recorded. Informed consent was obtained prior to both RB staining and scalpel biopsy procedures from each patient.

Based on clinical findings, scores were given as: Score 0- No clinical finding but H/O tobacco chewing habit; Score 1- Tobacco Induced Keratosis; Score 2-Homogenous Leukoplakia; Score 3- Erythroplakia; Score 4- OLP/ OLR/ OSMF/ Verrucous Leukoplakia.

RB stain was applied in area of tobacco quid placement. Clinical pictures were taken before and after staining. The area with highest stain uptake was selected for biopsy and incisional biopsy was carried out under Local Anaesthesia. Specimens were sent for histopathological examination. The intensity of staining was matched with shade guide using Adobe Photoshop CS3.

Shade tabs in the present study were made of filter paper which was stained by RB dye in gradient concentrations at room temperature for 1 hour $1 \%, 0.5 \%, 0.25 \%, 0.125 \%$ respectively. The grading was done as per the shade guide as: Grade I -

$0.125 \%$ shows $60-70 \%$ of magenta; Grade II $-0.25 \%$ shows $70-80 \%$ of magenta; Grade III - $0.5 \%$ shows 80 $90 \%$ of magenta; Grade IV - $1 \%$ shows $90-100 \%$ of magenta.

Histopathological grading was done as per Waldron criteria ${ }^{4}$ Grade 0- No stain; Grade 1- Hyperkeratosis; Grade 2- Mild Dysplasia; Grade 3- Moderate Dysplasia; Grade 4- Severe Dyspasia / OLP/ OLR / OSMF.

Among 105 patients, 48 patients were clinically diagnosed as Tobacco Induced Keratosis, 26 as Homogenous Leukoplakia, 8 as Erythroplakia, 8 as Oral Lichenoid Reaction, 2 as Oral Lichen Planus, 5 as Oral Submucous Fibrosis, 7 as clinically apparent lesion, 1as Verrucous Leukoplakia.

In the present study, 48 patients who were clinically diagnosed as Tobacco Induced Keratosis were in the age range of 20-29 years and is showing a shift in prevalence towards younger ages due to early exposure to tobacco.

26patients who were clinically diagnosed as Homogenous Leukoplakia were in the age range of 40-60 years. From the present study it was distinctly observed that oral leukoplakia was more prevalent in the age range of 51-60 years $(42 \%)$ followed by $41-50$ years $(28 \%)$ and $31-40(22 \%)$. This data is consistent with the findings of 
More C B et al 2011 who observed that oral leukoplakia was more prevalent in the age group of 55-60 years $(18.83 \%)$ followed by $45-49$ years $(16.23 \%)$ and $50-54$ years( $12.26 \%)$.

Therewas no significant difference in the mean duration of tobacco use among the four groups. This data is consistent with the findings of Renato Hopp et al ${ }^{5}$ in 2011, who observed that duration of tobacco consumption was similar among subjects with Oral Leukoplakia, Oral Cancer and tobacco consuming males.

In the present study, 7 patients among the total 105 cases had 'no' clinically evident lesion even with positive history of chewing habit. In these cases, staining was positive in areas of oral cavity where tobacco quid was placed. In a study conducted by Gu Fei Du et al, there were 25 false positive results among the total of 128 cases, which was due to visual examination error.

In the present study, 48 patients were clinically diagnosed as Tobacco Induced Keratosis. On staining, all the lesions in these patients took up the stain. Grade I staining was seen in 36 cases and Grade II staining in 12 cases. Gu Fei Du et al reported, 10 cases of Leukokeratosis, of which 5 revealed Grade I staining, 4 revealed Grade II staining, and 1 revealed Grade III staining.

In the present study, 26 patients were clinically diagnosed as Homogenous Leukoplakia, among whom 13 revealed Grade I staining, 12 revealed Grade II staining and 1 revealed Grade III staining. Gu Fei Du et al reported, 31 Leukoplakia patients, in which 5 revealed Grade I staining, 16 revealed Grade II staining, 10 revealed Grade III staining which was consistent with our study.

In the present study, on clinical examination, 8 patients were diagnosed with Erythroplakia and all revealed Grade III staining. 2 patients with Oral Lichen Planus, revealed Grade II and Grade III staining in each patient respectively. 8 pateints with Oral Lichenoid Reaction among whom 6 revealed Grade II and 2 revealed Grade III staining respectively. 5 patients with Oral Submucous Fibrosis and all revealed Grade I staining. 1 patient clinically diagnosed as Verrucous Leukoplakia and revealed Grade II staining.

Inference is that as lesions of keratosis and OSMF revealed milder degree of staining (ie Grade I) as compared to Erythroplakia which revealed Grade III staining indicating that simple keratosis may have less dysplastic features as compared to Erythroplakia where the degree of dysplasia is more.

All the cases were subjected to histopathological evaluation and the histopathological report (degree of dysplasia) was compared with shade grades. 7

patients with 'no' clinically evident lesion, were histopathologically reported as hyperkeratosis.

Among 48 pateints with Tobacco Induced keratosis, histopathologically 44 were reported as Hyperkeratosis and 4 as Mild Dysplasia. Gu Fei Du et al reported, 10 patients of Leukokeratosis among whom histopathologically 8 cases were reported as Hyperplasia, 1 as Chronic inflammation and 1 as Dysplasia.

In the present study, out of 26 patients with Homogenous leukoplakia, histopathologically 15 cases were reported as hyperkeratosis, 10 as mild and 1 as moderate dysplasia respectively. Gu Fei Du et al reported 31 Leukoplakia patients among whom 23 revealed Hyperplasia, 3 Dysplasia and 3 Chronic inflammation respectively and Squamous cell Carcinoma was reported in 2 cases, and is consistent with our study.

Clinical observations were substantiated histopathologically as it was noted that as the grade of staining of RB increased, the grade of dysplasia also increased.

In our study, sensitivity of RB stain was $93.3 \%$ and it is consistent with the study conducted by Gu Fei Du et al. As all lesions stained positive in our study with varying degree of intensity, hence specificity cannot be calculated. In the study conducted by Gu Fei Du et al, false negative results were seen in 2 patients (1.6\%) with OSCC. False positive results were also seen in 25 patients which could be attributed to error in visual examination. To overcome this limitation, in our study, after staining, clinical picture was taken and transferred 
to ADOBE PHOTOSHOP CS3 in which the degree of intensity of stain was estimated and compared with the varying degree of shades of RB stain concentration. This appears to be a reliable and accurate method.

In recent advances, fluorescence spectroscopic diagnostic method using RB stain has been developed in an animal model to make a quantified precancer diagnosis by Lei Zhang et al. ${ }^{6}$ The sensitivity and specificity to detect precancerous lesions is $100.00 \%$ and $87.50 \%$, respectively, when compared with scalpel biopsy. Thus the spectrofluorometric method mediated by RB could accurately discriminate different precancer stages, with low toxicity, noninvasiveness, and low cost, which constitutes a better alternative to toluidine blue staining for large scale oral cancer screening of high-risk patients. However, biopsy remains the final check for conclusive diagnosis of suspicious lesions (stages III and IV).

Limitations of the present study were small sample size, and only the subjects reporting to our institution were included which could lead to possibility of selection bias. Hence further studies with larger sample size need to be carried out within the community as a screening method to substantiate the results.

\section{Conclusion:-}

In the present study RB stain was taken up in all tobacco chewers with or without lesions. Sensitivity was $93.33 \%$ and the intensity of staining was directly proportional with the grade of dysplasia with highly significant p- value of $0.00001^{*}$

Studies with larger sample size need to be conducted to further validate the findings of the present study. Based on this study future research can be carried out to investigate the efficacy of Rose Bengal stain by using fluorescence spectroscopy in detecting precancerous lesions. The advantage of RB staining is that it is not taken up by inflammatory cells unlike Toluidine Blue stain. Thus to conclude, RB stain is simple, cost- effective, nonallergic and can be used as a diagnostic aid in the screening of potentially malignant disorders.

\section{Bibliography:-}

1. Bartsch H, Rojas M, Nair U, Nair J, Alexandrov K. Genetic susceptibility and DNA adults: Studies in smokers, tobacco chewers and coke oven workers. Cancer Detection and Prevention 1999; 23: 445.

2. Mittal, Palaskar, Shankari- Rose bengal staining- diagnostic aid for potentially malignant and malignant disorders : A pilot study. Indian journal of dental research 23(5), 2012.PAGE NO 561

3. Ge -fei-Du , $\mathrm{CZ} \mathrm{Li} \mathrm{,} \mathrm{HZ} \mathrm{Chen,} \mathrm{Xiao} \mathrm{Q,} \mathrm{Cao} \mathrm{Zg} \mathrm{et} \mathrm{al.} \mathrm{Rose} \mathrm{Bengal} \mathrm{staining} \mathrm{in} \mathrm{detection} \mathrm{of} \mathrm{oral}$ precancerous and malignant lesions with colorimetric evaluation- A pilot study. Int J Cancer 2007: 120:1958-1963

4. Waldron CA, Shafer WG. Leukoplakia revisited. Cancer 1975;36:1386-1392.

5. International Institute for Population Sciences (IIPS) and Ministry of Health and Family Welfare (MoHFW).Global adult tobacco survey (GATS) India 2009-2010. Mumbai and New Delhi: IIPS and MoHFW, Government of India; 2010.

6. Lei Zhang \& Liangjia Bi \& Jinna Shi \& Zhiguo Zhang \& Wenwu Cao \& Jiang Lin \& Chengzhang Li \& Jiarui Bi \& Yang Yu A quantitative diagnostic method for oral mucous precancerous is by Rose Bengal fluorescence spectroscopy Lasers Med Sci (2013) 28:241-246 DOI 10.1007/s10103-012-1054-y. 